\title{
SINCRONIA DE FLORAÇÃO ENTRE LANTANA CAMARA L. (VERBENACEAE) E PSITTACANTHUS CALYCULATUS (DC.) G. DON (LORANTHACEAE) OCORRENTES NAS DUNAS DE LA MANCHA, VERACRUZ, MEXICO
}

\author{
Mariluza Granja Barros \\ Herbário, Departamento de Botânica-IB \\ Universidade de Brasília \\ Caixa Postal 04457 \\ 70910-900 Brasília, DF. Brasil \\ Victor Rico-Gray \\ E \\ Ceecilia Díaz-Castelazo \\ Departamento de Ecologia Vegetal \\ Instituto de Ecología, A.C. \\ Apartado postal 63 \\ 91070 Xalapa, Veracruz, México
}

\begin{abstract}
RESUMO
Lantana camara L. e Psittacanthus calyculatus (DC.) G. Don, ocorrem simpatricamente e florescem em sincronia em La Mancha, Veracruz, México. Foram investigadas as estratégias florais (morfologia, coloração, recompensas) de cada espécie e o comportamento dos visitantes florais para se avaliar até que ponto a sincronia de floração interfere na eficiência de polinização. $L$. camara é uma espécie arbustiva, freqüente, ocorre formando densas touceiras, floresce praticamente durante o ano todo e apresenta vistosas inflorescências corimbosas. $P$. calyculatus é uma espécie hemiparasita, lenhosa, floresce de julho a novembro com densas inflorescências conspícuas. Ambas as espécies têm flores produtoras de néctar, tubulosas, de cor amarelo-alaranjada ao desabrochar, mudando para o vermelho-alaranjado no segundo dia. Devido à maior freqüência às flores de $L$. camara e à eficiência na coleta de pólen, os lepidópteros Agraulis vanillae, Anartia fatima, A. jatrophea e Junonia sp. foram considerados polinizadores primários enquanto que os beija-flores Amazilia yucatanensis, $A$. tzacatl foram considerados polinizadores primários de $P$. calyculatus. Doze espécies de lepidópteros visitaram indistintamente $L$. camara e $P$. calyculatus, tendo sido denominados polinizadores secundários. Com relação aos sistemas reprodutivos, os testes demonstraram que $L$. camara e $P$. calyculatus são xenogâmicas e auto-incompatíveis. Os dados obtidos, sobre a floração convergente, a similaridade na coloração floral e a síndrome de polinização de $L$. camara e $P$. calyculatus, sugerem que essas espécies estão envolvidas em um tipo de associação floral, no qual as duas beneficiam-se igualmente.
\end{abstract}

Palavras-chave: biologia da reprodução, fenologia floral, simpatria. 


\section{RESUMEN}

Lantana camara L. y Psittacanthus calyculatus (DC.) G. Don conviven simpátricamente y florecen al mismo tiempo en La Mancha, Veracruz, México. Se investigaron las estrategias de las flores (morfología, coloración, recompensas) de cada una de estas plantas y el comportamiento de los visitantes, para evaluar si la sincronía de la antesis interfiere en la eficiencia de la polinización. $L$. camara es una especie arbustiva, frecuente, encontrada espontáneamente en densos grupos; florece prácticamente durante todo el año y presenta bellas inflorescencias corimbosas. $P$. calyculatus es una planta hemi-parásita, leñosa, generalista, cuyas flores abren de julio a noviembre y sus triadas se agrupan en tirsos. Ambas especies tienen corolas tubulares, de color amarillo-anaranjado en antesis, cambiando a rojo-anaranjado al segundo día y producen néctar. Debido a su mayor frecuencia y eficiencia en la colecta del polen, los lepidópteros Agraulis vanillae, Anartia fatima, A. jatrophea y Junonia sp., fueron considerados polinizadores primarios de las flores de $L$. camara. Mientras que los colibríes Amazilia yucatensis y A. tzacatl se definieron en el mismo papel para $P$. calyculatus. Doce especies de lepidópteros que visitaron ambas especies se conceptuaron como polinizadores secundarios. Con relación a los sistemas reproductivos, las observaciones mostraron que L. camara y $P$. calyculatus son xenogámicas y auto-incompatibles. De acuerdo con los datos obtenidos sobre la floración convergente, la similitud en la coloración floral y el síndrome de polinización de Lantana camara y Psittacanthus calyculatus, se sugiere que esas especies están involucradas en un tipo de asociación floral en el cual las dos se benefician igualmente.

Palabras clave: biología de la reproducción, fenología floral, simpatría.

\section{ABSTRACT}

Lantana camara L. and Psittacanthus calyculatus (DC.) G. Don occur sympatrically and flower synchronously at La Mancha, Veracruz, Mexico. Floral strategies as morphology, colour and rewards, as well as visitors behaviour, were investigated to evaluate if floral synchrony influences pollination efficiency. L. camara is a common shrub, which occurs in clumps, flowers throughout the year, and displays showy corymbose inflorescences. $P$. calyculatus is a woody hemiparasite, with conspicuous inflorescences, that flowers from July to November. Both species are nectar producers, have flowers with tubular corollas, which are yellow-orange at anthesis, turning to reddish-yellow when fading. Because of their frequency and the ability to contact the sexual organs, as main (primary) pollinators of L. camara are considered the lepidopteran Agraulis vanillae, Anartia fatima, A. jatropha and Junonia sp.; whereas the hummingbirds Amazilia yucatensis and $A$. tzacatl, are suggested as primary pollinators of $P$. calyculatus. Twelve species of lepidopterans which visited flowers of both species were considered as secondary pollinators. Breeding systems tests revealed that $L$. camara and $P$. calyculatus are xenogamous and self-incompatible. Data from floral convergence, floral colour similarities and pollination syndromes suggest that these species are involved in a floral association in which both have equal benefit.

Key words: reproductive biology, floral phenology, sympatry.

\section{INTRODUÇÃO}

Os estudos que investigam as interações existentes entre as plantas e os animais são de grande importância para o entendimento das estratégias de co-evolução desenvolvidas entre os dois grupos. Nos trópicos, os estudos sobre a ocorrência de espécies vegetais 
simpátricas com floração convergente têm se reportado a congenéricos como em Heliconia (Stiles, 1975), Costus (Schemscke, 1981), Dalechampia (Armbruster y Herzig, 1984), Aphelandra (Mc Dade, 1984), Styrax (Saraiva et al., 1988), Angelonia (Vogel y Machado, 1991), Vochysia (Oliveira y Gibbs, 1994), Byrsonima, Diplusodon e Erythroxylum (Barros, 1992, 1996, 1998). Entretanto, pouco tem sido documentado sobre as relações de simpatria e a ecologia da polinização entre gêneros de famílias diferentes que apresentam similaridade na morfologia floral e florescem sincronicamente. No Brasil, os estudos de Noronha y Gottsberger (1980) com Aspilia floribunda (Asteraceae) e Cochlospermum regium (Cochlospermaceae), e os de Yanagizawa y Gottsberger (1983) com Distictella elongata (Bignoniaceae) e Crotalaria anagyroides (Fabaceae) foram os únicos encontrados nesta linha de pesquisa.

Para este estudo, foram selecionadas Lantana camara L. (Verbenaceae), uma espécie arbustiva e Psittacanthus calyculatus (DC.) G. Don (Loranthaceae), uma espécie lenhosa hemiparasita, que ocorrem simpatricamente nas dunas de La Mancha, Veracruz, México, florescem simultaneamente e são frequentemente visitadas pelos mesmos vetores de polinização. Este trabalho tem por objetivos: comparar as estratégias florais e a ecologia de polinização dessas duas espécies para entender como a sincronia de floração interage na eficiência de polinização de ambas. Esta pesquisa é pioneira na investigação da sincronia de floração em $L$. camara e $P$. calyculatus.

\section{METODOLOGIA}

O trabalho de campo desenvolveu-se de setembro a dezembro de 1996, no Centro de Investigaciones Costeras de La Mancha (CICOLMA), Veracruz, México $\left(96^{\circ} 22^{\prime} \mathrm{W}\right.$, $19^{\circ} 36^{\prime} \mathrm{N}$; altitude < $110 \mathrm{~m}$, de acordo com Garcia-Franco y Rico-Gray, 1995). A Reserva possuiu cerca de 70 ha, com precipitação média de 1100 a 1300 mm/ano, temperatura média de $26^{\circ}$ a $28^{\circ} \mathrm{C}$, com a mínima de $10^{\circ} \mathrm{C}$ e a máxima de $38^{\circ} \mathrm{C}$ (segundo MorenoCasasola, 1982). A vegetação é formada por comunidades distintas, composta por florestas tropicais decíduas, florestas tropicais secas, dunas costeiras, lagoas e pântanos (Rico-Gray y Lot, 1983; Fragoso et al., 1994), que interagem formando um mosaico dinâmico (MorenoCasasola et al., 1982, Kellman y Roulet, 1990, apud Ortiz-Pulido et al., 1995). As diferentes fitofisionomias favorecem à diversidade florística, tendo sido documentadas 313 espécies incluíndo dados de herbário (Guerrero, 1984). As dunas de La Mancha são estáveis com matorrales (vegetação agregada), têm rica diversidade florística como documentado por Moreno-Casasola et al. (1982).

$P$. calyculatus e $L$. camara apresentaram alto índice de frequência nas dunas de la Mancha, sendo que $P$. calyculatus ocorre abundantemente em plantas da família Leguminosae. A área de estudo compreendeu cerca de $1.5 \mathrm{~km}$ das dunas com vegetação lenhosa, principalmente arbórea e arbustiva. Foram observados na área de estudo, cerca de 90 indíviduos (manchas, touceiras) de cada espécie. Foram registrados dados em herbários (MEXU, UB, XAL), sobre a época de floração e frutificação das duas espécies, para posterior comparação com aqueles obtidos no campo.

Lantana camara é uma espécie arbustiva originária da América tropical, sendo encontrada espontaneamente em áreas subtropicais, sua floração ocorre praticamente durante o ano todo e a frutificação também é contínua (Castillo y Carabias, 1982). 
Psittacanthus calyculatus é uma espécie hemiparasita, lenhosa, encontrada nos trópicos, ocorre em hospedeiros arbóreos e segundo García-Franco et al. (1995), é generalista, sendo documentada associada a várias espécies distintas. De acordo com Castillo y Carabias (1982), sua frutificação é irregular durante o período chuvoso.

Para este estudo foram marcados quatro indivíduos hospedeiros de $P$. calyculatus, sendo três de Diphysa robinioides e um de Acacia macracantha, todos da família Leguminosae. O número exato dos plantas de $P$. calyculatus estabelecido em cada hospedeiro tornou-se difícil de precisar, devido ao emaranhado de ramos e subdivisões dos mesmos. Por isso, o "conjuntos de ramos" em cada hospedeiro foi considerado como um indivíduo. Sendo delimitado da seguinte maneira: Diphysa robinioides $\mathrm{n}^{\circ}$ 1, com quatro indivíduos de $P$. calyculatus, $\mathrm{n}^{\circ}$ 2, com cinco indivíduos e ํㅜ 3, com cinco indivíduos; em Acacia macracantha foram marcados três indivíduos de $P$. calyculatus. Para a marcação dos indivíduos de L. camara, foram usados aqueles que circundavam os hospedeiros de $P$. calyculatus. Os espécimes de $L$. camara apresentam-se fortemente esgalhados com ramos entrelaçados, formando touceiras (manchas de vegetação) bem delimitadas. Este hábito de crescimento dificultou a acuridade da contagem, de modo que cada touceira foi considerada como um indivíduo, (oito no total), sendo que cada um tinha cerca de $1.2 \mathrm{~m}$ de circunferência.

Exemplo de marcação dos indivíduos:

\begin{tabular}{lcccccc} 
& Diphysa & robinioides & Acacia macracantha & Total \\
\hline Indivíduos & 1 & 2 & 3 & 1 & \\
\hline$P$. calyculatus & 4 & 5 & 5 & 3 & 17 \\
L. camara & 1 & 3 & 2 & 2 & 8
\end{tabular}

Foram anotados dados de campo referentes a fenologia da floração e frutificação das duas espécies, número de flores/inflorescência, assim como, sobre as estratégias das flores, incluindo: arquitetura floral, duração da flor, horário de antese, concentração de sacarose (usando-se refractômetro de bolso - Bellingham \& Stanley). Para a contagem das flores por inflorescência, foram selecionadas ao acaso 5 inflorescências de cada espécie (Tabela 1). Para a documentação da duração das flores e antese, foram etiquetados cerca de 10 botões florais de cada espécie, tendo sido acompanhado o desabrochamento (antese) de cada flor até a senescência. Para a medição da concentração de sacarose e quantidade de néctar, foram ensacados dez botões florais de cada espécie, escolhidos aleatoriamente. A medição foi feita nos dias 25 e 26 de setembro, das 9 h00 às 11 h00. Algumas flores das duas espécies foram coletadas e conservadas em álcool a 70\%, para a análise das peças florais no laboratório (Tabela 2, Pranchas 1 e 2). Foi registrado o comportamento dos visitantes florais, incluindo horário de visita, permanência na flor, possibilidade de contato com os órgãos reprodutivos das flores, habilidade na coleta do néctar e eficiência na retirada do pólen. Foram considerados polinizadores primários, os visitantes mais freqüentes que foram observados contactando os verticilos reprodutivos e que apresentavam grande carreamento de polén no corpo. Foram considerados polinizadores secundários aqueles 
menos freqüentes, porém que também contactavam os verticilos reprodutivos e carreavam alguma quantidade de polén. A freqüência foi estipulada pelo número de visitas, por minuto. Quando possível, os visitantes florais foram capturados, para posterior identificação (Tabela 3, Prancha 3). Para avaliar os sistemas reprodutivos, foram feitos três testes de polinização artificial para a observação da formação de frutos: 1) autopolinização induzida - flores foram ensacadas em pré-antese, polinizadas (com o próprio pólen) durante a antese e reensacadas; 2) autopolinização automática - flores foram só ensacadas em pré-antese; e 3) polinização natural (controle) - flores foram só etiquetadas (Tabela 4). Algumas flores pós-polinizadas, foram coletadas para posterior observação ao microscópio de fluorescência, usando-se a técnica de Martin (1959). As outras espécies que estavam em floração simultânea, foram anotadas para comparações fenológicas posteriores.

\section{RESULTADOS}

Fenologia de floração

De acordo com os dados de herbário, foi constatado que no México Lantana camara floresce de janeiro a dezembro coincidindo com sua floração no Brasil (UB), e Psittacanthus calyculatus de julho a novembro. Durante o período em que foi acompanhada a fenologia de floração de $L$. camara e $P$. calyculatus, foi constatado que as duas espécies floresceram abundantemente, sendo que em setembro a floração de ambas foi intensa, começando a declinar a partir da primeira semana de outubro. Com relação à convergência floral de $L$. camara e $P$. calyculatus sugere-se que esta deve incrementar a taxa de visitação, devido ao número de flores por inflorescência (Tabela 1).

Tabela 1. Comparação do número de flores por inflorescência $(n=5)$.

\begin{tabular}{|lcccccc|cccccc|}
\hline \multicolumn{7}{c|}{ Setembro 25 } & \multicolumn{5}{c|}{ Outubro 15 } \\
\hline Inflorescências & 1 & 2 & 3 & 4 & 5 & Total & 1 & 2 & 3 & 4 & 5 & Total \\
\hline L. camara & 38 & 40 & 32 & 36 & 37 & 183 & 28 & 27 & 27 & 26 & 27 & 135 \\
$P$. calyculatus & 55 & 56 & 42 & 40 & 43 & 236 & 22 & 23 & 26 & 25 & 30 & 126 \\
\hline
\end{tabular}

Morfologia floral

Os dados comparativos da morfologia floral de $L$. camara e $P$. calyculatus demonstram algumas similaridades existentes entre as duas espécies, com relação à sincronia floral, coloração das flores, produção de néctar, horário da antese e duração da flor (Tabela 2). Em L. camara, as flores recém-abertas são amarelo-forte, desabrocham do raio para o centro (centrípeta) a partir das $6 \mathrm{~h} 30$, exsudam néctar e no segundo dia, tornamse vermelho-alaranjadas, sem néctar. A corola é tubular-estreita, os estames são inclusos, 
adnados à corola; o ovário contém um ou dois óvulos e o estigma é globoso (Prancha 1). Em $P$. calyculatus, as flores desabrocham com uma coloração amarelo-alaranjada, contrastando com vermelho-alaranjado dos verticilos reprodutivos, sendo que a coloração fica mais forte a partir do segundo dia. As flores recém-abertas (7h00), exsudam néctar e as do segundo dia têm néctar com concentração mais alta de sacarose. A corola simula uma forma tubular (aparentemente as pétalas são soldadas na base); os estames são epipétalos, originam-se na porção mediana do tubo corolíneo e junto com esse ofrecem um "campo de pouso" para os visitantes; o ovário se apresenta com a base imersa em uma glândula nectarífera e o estilete é reto (Prancha 2). A mudança de coloração nas flores do segundo dia de $L$. camara e $P$. calyculatus, tornando-se mais escuras, foi interpretada como uma estratégia de atração para os polinizadores, devido ao fato de que as inflorescências seriam visualizadas mais facilmente, oferecendo melhor orientação aos mesmos.

Tabela 2. Comparação das estratégias florais de Lantana camara e Psittacanthus calyculatus $(\mathrm{n}=12)$.

\begin{tabular}{|l|l|l|}
\hline Estratégias florais & Lantana camara & Psittacanthus calyculatus \\
\hline Inflorescência & corimbosa & tirso com flores em tríades \\
Número de flores por inflorescência & 26 a 40 & 22 a 56 \\
Concentração de sacarose (néctar) & ${ }^{*} 28 \%(\mathrm{n}=10)$ & ${ }^{*} 30 \%,{ }^{* *} 32 \%(\mathrm{n}=5)$ \\
Quantidade (néctar) & $0.4 \mu 1$ & $1.8 \mu 1$ \\
Horário de antese & $6 \mathrm{~h} 30$ até às $15 \mathrm{~h} 00$ & $7 \mathrm{~h} 00$ às $16 \mathrm{~h} 00$ \\
Viabilidade da flor & 1 dia & 1 dia \\
Duração da flor & 2 dias & 2 dias \\
\hline
\end{tabular}

*flores do primeiro dia, **flores do segundo dia

Vetores de polinização

As flores de $L$. camara e $P$. calyculatus, foram visitadas conjuntamente por 13 espécies de lepidópteros e por quatro de himenópteros. Destes as abelhas Trigona sp., Augochloropsis sp., Apis mellifera e Trigonisca sp. foram consideradas pilhadoras de pólen devido ao fato de não terem sido observadas efetuando a polinização. Os lepidópteros Agraulis vanillae (Prancha 3), Anartia fatima, A. jatrophea e Junonia sp. foram considerados polinizadores primários de $L$. camara, devido à maior freqüência às flores e à eficiência na coleta de pólen, enquanto que os beija-flores Amazilia yucatanensis e A. tzacatl foram considerados polinizadores primários de $P$. calyculatus. Cynanthus latirostris (Trochilidae) também visitou as flores de $P$. calyculatus, porém que foi considerado pilhador, por não ter sido observado contatando os órgãos sexuais de $P$. calyculatus. Os polinizadores efetivos e ocasionais das duas espécies estão listados na Tabela 3. A arquitetura da inflorescência de L. camara parece corresponder à arquitetura de uma flor de $P$. calycultus. Isto é, ambas oferecem similar campo de pouso aos lepidópteros. O fato de que as duas espécies apresentam peças florais com tamanhos diferentes, possivelmente não 
compromete o transporte do pólen, que poderá ser levado em diferentes locais dos corpos dos animais. O transporte do pólen de $P$. calyculatus por lepidópteros, foi feito nos pêlos do corpo e nas escamas das asas, e de $L$. camara na região próxima à probócide. Em L. camara a polinização ocorre durante o desenrolar e enrolar da probócide, dentro do tubo corolíneo, que é muito estreito e em $P$. calyculatus durante o pouso desses vetores na corola. A duração da visita dos lepidópteros é breve, ca. de 2 a $3 \mathrm{seg}$. Os beija-flores que visitaram as flores de $P$. calyculatus, aterrissavam muito rapidamente, demonstrando que "sabiam" como coletar o néctar. As visitas foram rápidas, ca. de 2 seg., sugerindo a possibilidade de que o pólen era transportado nas imediações do bico. Tanto os lepidópteros com os beija-flores percorriam muitas flores de uma mesma inflorescência durante uma viagem de forrageamento e visitavam mais de uma vez as mesmas flores. As flores do primeiro das duas espécies foram visitadas preferencialmente, não tendo sido observada nenhuma preferência por flores do segundo dia.

Tabela 3. Vetores de polinização em Lantana camara e Psittacanthus calyculatus $(x=$ polinizadores primários, y = polinizadores secundários).

\begin{tabular}{|c|c|c|}
\hline Vetores de polinização & L. camara & P. calyculatus \\
\hline \multicolumn{3}{|l|}{ Lepidoptera } \\
\hline \multicolumn{3}{|l|}{ Nymphalidae } \\
\hline Agraulis vanillae & $x$ & $\mathrm{y}$ \\
\hline Anartia fatima & $x$ & - \\
\hline A. jatrophae & $x$ & - \\
\hline Dryas iulia cillene & $y$ & y \\
\hline Euptoieta hegesia & y & y \\
\hline E. claudia & y & y \\
\hline Junonia sp. & $x$ & - \\
\hline \multicolumn{3}{|l|}{ Papilionidae } \\
\hline Battus belus & $\mathrm{y}$ & y \\
\hline B. polydamus & $y$ & y \\
\hline Heraclides anchisiades & $y$ & y \\
\hline Papilio polyxenes & $y$ & $y$ \\
\hline \multicolumn{3}{|l|}{ Pieridae } \\
\hline Ascia monuste & $y$ & $y$ \\
\hline Phoebis argante & $y$ & y \\
\hline Phoebis sp. & $y$ & $y$ \\
\hline Urbanus sp.1 & $y$ & $y$ \\
\hline Urbanus sp.2 & $y$ & y \\
\hline \multicolumn{3}{|l|}{ Passeriformes } \\
\hline \multicolumn{3}{|l|}{ Trochilidae } \\
\hline Amazilia yucatanensis & - & $x$ \\
\hline A. tzacatl & - & $x$ \\
\hline
\end{tabular}




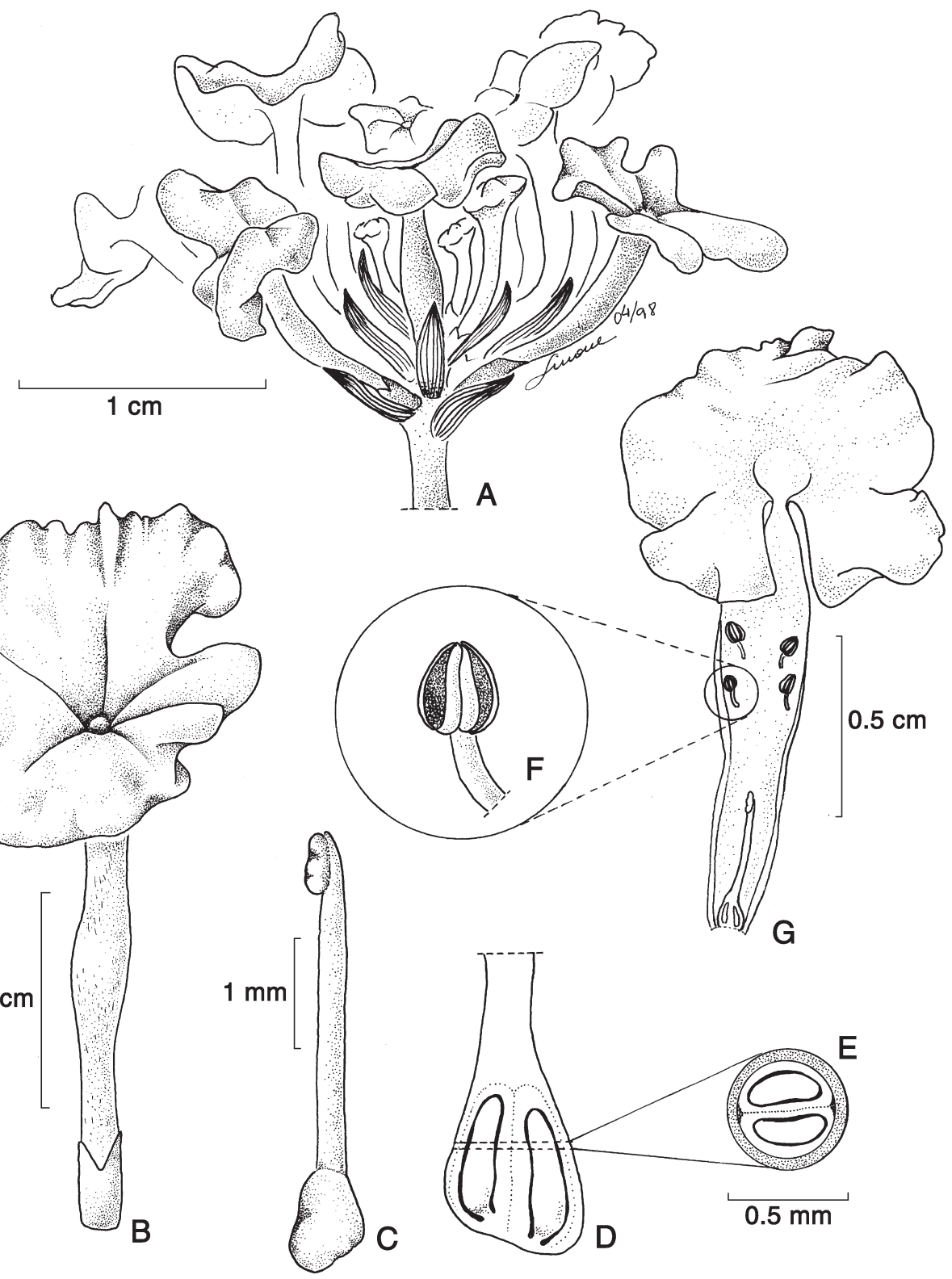

Prancha 1. Lantana camara: A. Inflorescência; B. Flor; C. Gineceu; D e E. Ovário em corte longitudinal e transversal; F. Antera; G. Inserção da antera no tubo da corola. 
Granja et al.: Sincronia de Floracão nas Dunas de La Mancha, Veracruz
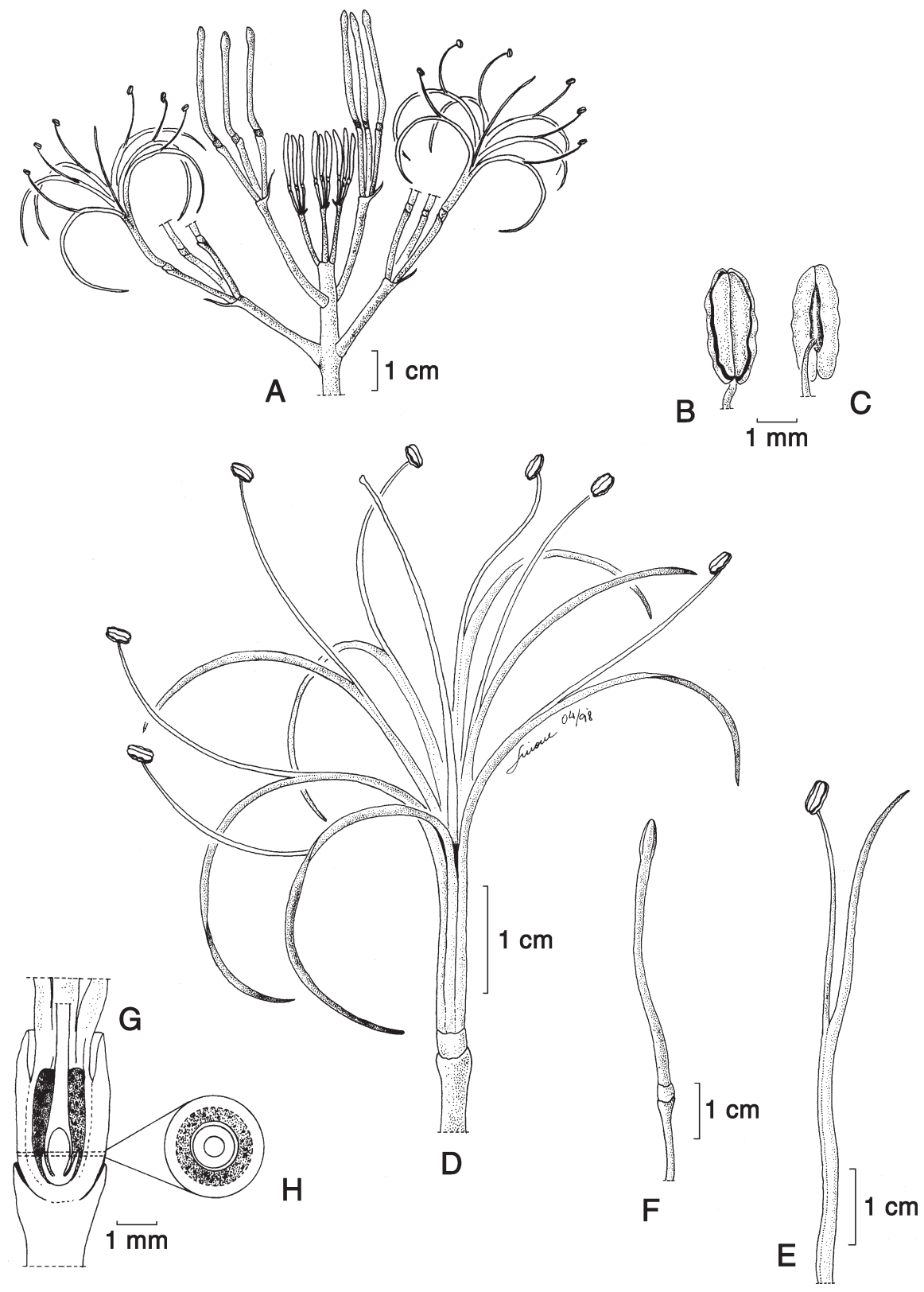

Prancha 2. Psittacanthus calyculatus: A. Inflorescência; B e C. Antera; D. Tubo corolíneo; E. Adjunção do estame à pétala; $\mathrm{F}$. Botão floral; $\mathrm{G}$ e H. Ovário em corte longitudinal e transversal. 


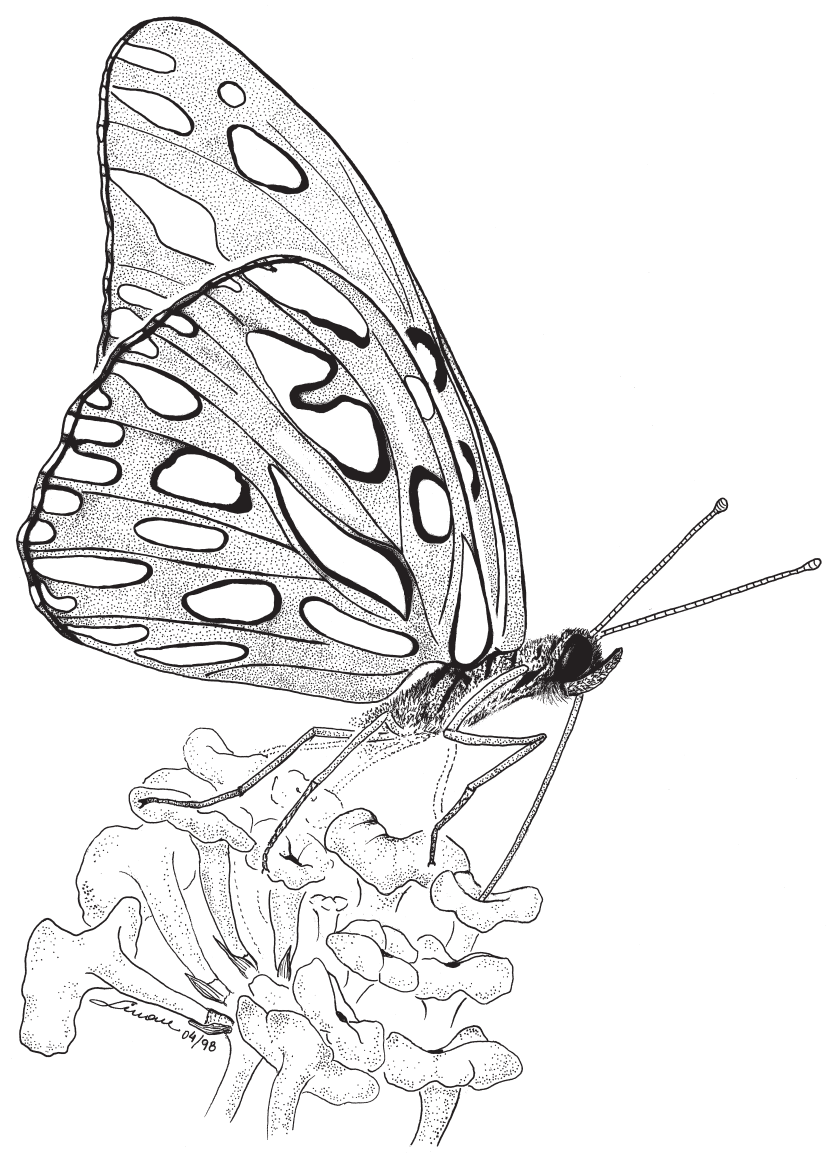

Prancha 3. Agraulis vanillae (Nymphalidae) coletando néctar em Lantana camara.

\section{Biologia Reprodutiva}

Conforme os dados apresentados na Tabela 4, constatou-se que $L$. camara e $P$. calyculatus, são auto-incompátiveis com frutos formados resultantes só de polinização natural. Em L. camara foram formados $49.2 \%$ de frutos e em P. calyculatus $23.3 \%$. Para ambas espécies, do estudo dos pistilos pós-polinizados ao microscópio de fluorescência (24h00, 48h00 e 72h00), constatou-se tubos polínicos sendo bloqueados no estigma e na porção superior do estilete em $L$. camara. Em $P$. calyculatus foram detectados tubos polínicos até na parte apical do ovário. Isto foi confirmado por sinais de engrossamento dos ovários de algumas flores, com aproximadamente uma semana de auto-polinizadas. Em geral, L. camara formou de 1 a 13 frutos por inflorescência (média 6.6), sendo que cerca de $40 \%$, estavam sendo danificados por vespas. 
Granja et al.: Sincronia de Floracão nas Dunas de La Mancha, Veracruz

Tabela 4. Testes dos sistemas reprodutivos de Lantana camara e Psittacanthus calyculatus.

\begin{tabular}{|l|c|c|}
\hline Testes & L. camara & $P$. calyculatus \\
\hline & $\mathrm{n} / \mathrm{f} / \%$ & $\mathrm{n} / \mathrm{f} / \%$ \\
Autopolinização induzida & $21 /-/-$ & $48 /-/-$ \\
Autopolinização espontânea & $59 /-/-$ & $67 /-/-$ \\
Polinização natural & $65 / 32 / 49.2$ & $71 / 18 / 25.3$ \\
\hline
\end{tabular}

\section{DISCUSSÃO}

Espécies vegetais co-ocorrentes e pertencentes à famílias diferentes que florescem em sincronia, possivelmente devem competir por polinizadores, como documentado em Lantana camara e Psittacanthus calyculatus. De acordo com Schemske (1976), se a competição por polinizadores está ligada a coexistência de flores homólogas, esta pode ser prejudicial a capacidade reprodutiva das mesmas. No estudo em questão, a maior semelhança encontrada foi relativa à coloração das flores, que permitiu interpretar esse fato como um esforço conjunto das duas espécies para atrair um maior número de visitantes. Essas semelhanças foram relacionadas à presença de polinizadores secundários que certamente aumentam as chances da transferência do pólen, em ambas espécies. As plantas tornam a floração conspícua aos polinizadores específicos, diminuindo o gasto de energia e a distância entre as fontes alimentares (Heinrich, 1983). No caso de L. camara e $P$. calyculatus, ainda há a considerar a mudança de coloração nas flores, que tornamse mais escuras a partir do segundo dia. Esse fenômeno foi relacionado ao aumento de atratividade das inflorescências, o que facilitaria a visualização dos polinizadores. Mudanças nas colorações das flores têm sido associadas à maior frequência de polinizadores (e.g. thrips, de acordo com Mathur y Mohan-Ram, 1986), facilitando a orientação e ao mesmo tempo advertindo-os que as flores do segundo dia não têm mais recompensas, aumentando assim a eficiência de polinização e diminuindo o gasto energético dos mesmos (Waser, 1983; Weiss, 1991, 1995), como documentado em Quisqualis indica (Eisikowitch y Rotem, 1987), Lupinus argenteus (Gori, 1989) e Combretum fruticosum (Bernadello et al., 1994). Nesses casos, a permanência das flores mais velhas, foi ainda relacionada ao aumento do tamanho da inflorescência e à segurança da plataforma de pouso. Entretanto, Macarena Arenas (http://fig.cox.miami.edu.Programs/arenas. htm), estudando $L$. camara, documentou visitação também em flores mais velhas que tinham mudado de cor. $O$ fato de $L$. camara e $P$. calyculatus, repartirem os mesmos polinizadores secundários, sugere que a similaridade floral entre ambas é importante para manter o interesse dos mesmos, por pelo menos quatro razões: 1) incremento da atratividade; 2) os grãos de pólen são transportados em diferentes locais do corpo dos visitantes florais; 3) ambas as espécies têm um número reduzido de óvulos, e 4) aumento das possibilidades de recombinações genéticas, devido ao aumento das chances de doadores. No caso de polinizadores específicos, Agraulis vanillae tem sido reconhecida com polinizador efetivo de L. camara (Barrows, 1976; Weiss, 1991) e tem demonstrado preferência por flores amarelas (Weiss, 1995). No entanto, Macarena Arenas constatou que Apis mellifera apesar de ter uma probócide curta, conseguiu coletar néctar de L. camara que ocorria próximo 
a um apiário. Entretanto, a eficiência de polinização por borboletas em L. camara, parece não ser tão frequentemente documentada. Mathur e Mohan Ram (1986) identificaram thrips (Thysanoptera) como regular e eficiente polinizador de L. camara ocorrente na Índia. Por outro lado a polinização por Amazilia spp. tem sido documentada em espécies com síndrome de ornitofilia que apresentam flores tubulosas com coloração vistosa e néctar diluído (Baker y Baker, 1983), semelhantes às de $P$. calyculatus, como em Heliconia (Linhart, 1973), em Combretum (Bernadello et al., 1994) e em Palicourea padifolia (Ree, 1997).

Em cerrados brasileiros, sincronia de floração e estratégias florais semelhantes em espécies simpátricas que são visitadas pelos mesmos vetores, têm sido observado por um dos autores (M. Barros, observ. pessoal) como é o caso de: Trembleya parviflora (Melastomataceae) e Diplusodon virgatus (Lythraceae) ambas com flores alvas e produtoras de pólen; de Byrsonima laxiflora (Malpighiaceae) e Vochysia pyramidalis (Vochysiaceae) que têm flores amarelas, e também em Justicia lanstyakii (Acanthaceae) e Stachytarpheta schaueri (Verbenaceae) que apresentam flores tubulosas e vermelhas com síndrome de ornitofilia. No presente trabalho, também foi documentada convergência de floração em Chamaecrista chamaecristoides, Tecoma stans, Stylosanthes sp., Pectis satureoides, Turnera diffusa e T. ulmifolia, que também apresentam flores amarelas e foram comumente visitadas pelos mesmos lepidópteros de $L$. camara e $P$. calyculatus.

Com relação aos sistemas reprodutivos de $P$. calyculatus, é possível que essa espécie apresente late acting incompatibility. Isto é, que os tubos polínicos sejam bloqueados no ovário, como registrado por Seavey y Bawa (1986) para espécies tropicais. Este fenômeno, também foi observado em três espécies de Erythroxylum (Barros, 1998) e duas de Tabebuia que ocorrem em cerrados brasileiros (Barros, no prelo). Na comunidade estudada, a síndrome de polinização em que $L$. camara e $P$. calyculatus estão envolvidas, desempenha um papel fundamental para a polinização cruzada destas espécies. Para se aprofundar nos estudos das associações florais existentes entre ambas, testes de comparação geográfica, de taxas de visitação, dos eventos de floração e de frutificação e dos sistemas reprodutivos deverão ser realizados.

\section{AGRADECIMENTOS}

A primeira autora agradece à Dra. Mónica Palacios-Rios, pela identificação dos lepidópteros, ao Dr. José García-Franco, pela colaboração e ao Dr. Raúl Ortiz-Pulido, pela identificação das aves, todos do Instituto de Ecología, A.C. (Mexico); ao Dr. Tarciso Filgueiras IBGE/Brasil, pela leitura e sugestões ao manuscrito; aos Drs. Maurício Ayala e Paulo César Motta, da Universidade de Brasília (Brasil), pela versão do Resumo para o espanhol e pela identificação dos insetos, ao Carlos Frederico Barros pela preciosa ajuda no campo, ao CNPq/Brasil (process no 46051696/5-NV) pelo auxílio financeiro e ao Instituto de Ecología, A.C. (902-16), pelo suporte para o desenvolvimento das pesquisas de campo.

\section{LITERATURA CITADA}

Armbruster, W. S. y A. L. Herzig. 1984. Partioning and sharing of pollinators by four sympatric species of Dalechampia (Euphorbiaceae) in Panama. Annals of the Missouri Botanical Garden 71: 1-6. 
Baker. G. e I. Baker. 1983. Floral nectar sugar constituents in relation to pollinator type. In: Jones, C. E. \& R. J. Little (eds.) Handbook of experimental pollination biology. Scientific and Academic Editions. Nueva York. pp. 117-141.

Barros, M. A. G. 1992. Fenologia de floração, estratégias reprodutivas e polinização de espécies simpátricas do gênero Byrsonima Rich. (Malpighiaceae). Revista Brasileira de Biologia 52: 343-353.

Barros, M. A. G. 1996. Biologia reprodutiva e polinização de espécies simpátricas de Diplusodon (Lythraceae). Acta Botanica Mexicana 37: 11-21.

Barros, M. A. G. 1998. Sistemas reprodutivos e polinização de espécies simpátricas de Erythroxylum (Erythroxylacae). Revista Brasileira de Botânica 22: 159-166.

Barrows, E. M. 1976. Nectar robbing and pollination of Lantana camara (Verbenaceae). Biotropica 8: 132-135.

Bernadello, L., L. Galetto e I. G. Rodriguez. 1994. Reproductive biology, variability of nectar and pollination of Combretum fruticosum (Combretaceae) in Argentina. Botanical Journal of the Linnean Society 114: 293-308.

Castillo, S. y J. Carabias. 1982. Ecología de la vegetación de dunas costeras: fenologia. Biotica 7 : 551-568.

Eisikowitch, D. y R. Rotem. 1987. Flower orientation and color change in Quisqualis indica and their possible role in pollinator partioning. Botanical Gazette 148: 175-179.

Fragoso, C., B. Delfosse y P. Moreno-Casasola. 1994. Centro de Investigaciones Costeras de La Mancha. Instituto de Ecología, A.C. Xalapa, Veracruz, México. 2 pp.

García-Franco, J. G., V. Rico-Gray y M. Palacios-Rios. 1995. Parasitismo de Psittacnthus calyculatus (Loranthaceae) sobre Beaucarnea gracilis (Nolinaceae) en el valle de Zapotitlán de las Salinas, Puebla, México. Cactáceas y Suculentas Mexicanas 40: 62-65.

García-Franco, J. G. y V. Rico-Gray. 1995. Population structure and clonal growth in Bromelia pinguin L. (Bromeliaceae) in dry forests of coastal Veracruz, México. Tulane Studies in Zoology and Botany 30: 27-37.

Gori, D. F. 1989. Floral color change in Lupinus argenteus (Fabaceae): why should plants advertise the location of unrewarding flowers to pollinators? Evolution 43: 870-881.

Guerrero, B. 1984. Iventario florístico de la reserva ecológica el Morro de la Mancha. Instituto Nacional de Investigaciones sobre Recursos Bióticos, Xalapa, Veracruz, México. 35 pp.

Henrich, B. 1983. Insect foraging energetics. In: Jones, C. E. \& R. J. Little (eds.)Handbook of experimental pollination biology. Scientific and Academic Editions. Nueva York. pp. 187-214.

Kellman, M. y N. Roulet. 1990. Nutrient flux and retention in a tropical sand-dune succession. Journal of Ecology 78: 664-676.

Linhart, W. B. 1973. Ecologicial and behavioral determinants of pollen dispersal in hummingbirdpollinated Heliconia. American Naturalist 107: 511-523.

Martin, F. S. 1959. Staining and observing pollen tubes in the style by means of fluorescence. Stain Technology 34: 125-128.

Mathur, G. y H. Y. Moham-Ram. 1986. Floral biology and pollination of Lantana camara. Phytomorphology 36: 79-100.

McDade, L. 1985. Breeding systems of Central American Aphelandra (Acanthaceae). American Journal of Botany 71: 1515-1521.

Moreno-Casasola, P. 1982. Ecologia de la vegetacion de las dunas costeras: factores físcos. Biotica 7: $577-602$.

Moreno-Casasola, P., E. van der Maarel, S. Castillo, M. L. Huesca e I. Pisanty. 1982. Ecología de la vegetación de dunas costeras: estructura y composición en el Morro de La Mancha. Ver. I. Biotica 7: 577-602.

Noronha, M. R. P. y G. Gottsberger. 1980. A polinização de Aspilia floribunda (Asteraceae) e Cochlospermum regium (Cochlospermaceae). Revista Brasileira de Botânica 3: 67-77. 
Oliveira, P. E. y P. E. Gibbs. 1994. Pollination biology and breeding systems of six Vochysia species. Journal of Tropical Ecology 10: 509-522.

Ortiz-Pulido, R., H. Gomez de Silva, F. González-Garcia y A. Álvarez. 1995. Avifauna del Centro de Investigaciones Costeras de La Mancha, Veracruz, México. Acta Zoologica Mexicana (n. S.) 66: 87-118.

Ree, R. H. 1997. Pollen flow, fecundity, and the adaptive significance of heterostyly in Palicourea padifolia (Rubiaceae). Biotropica 29: 298-308.

Rico-Gray, V. y A. Lot. 1983. Producción de hojarasca del manglar de la Laguna de La Mancha, Veracruz, México. Biotica 7: 295-301.

Saraiva, L., O. César y R. Monteiro. 1988. Biologia da polinização e sistema de reprodução de Styrax camporum Pohl e S. ferrugineus Nees et Mart. (Styracaceae). Revista Brasileira de Botânica 11: $71-80$.

Schemske, D. W. 1976. Pollinator specificity in Lantana camara L. (var.) trifolia (Verbenaceae). Biotropica. 8: 260-264.

Schemske, D. W. 1981. Floral convergence and pollinator sharing in two bee-pollinated tropical herbs. Ecology 62: 946-954.

Seavey, S. R. y K. S. Bawa. 1986. Late acting self-incompatibility in angiosperms. Botanical Review 52: $196-215$.

Stiles, F. G. 1975. Ecology, flowering phenology and hummingbird pollination of some Costa Rican Heliconia species. Ecology 56: 285-301.

Vogel, S. e I. C. Machado. 1991. Pollination of four sympatric species of Angelonia (Scrophulariaceae) by oil-collecting in NE-Brazil. Plant Systematics and Evolution 178: 153-178.

Waser, N. M. 1983. Competition for pollination and floral character differences among sympatric plant species: a review of evidence. In: Jones, C. E. \& R. J. Little (eds.). Handbook of experimental pollination biology. Scientific and Academic Editions. Nueva York. pp. 277-293.

Weiss, M. R. 1991. Floral color change: a widespread functional convergence. American Journal of Botany 82: 167-185.

Weiss, M. R. 1995. Associative colour learning in a nymphalid butterfly. Ecological Entomology 20: 298-301.

Yanagisawa, Y. y G. Gottsberger. 1983. Competição entre Distictella elongata (Bignoniaceae) e Crotalaria anagyroides (Fabaceae) com relação às abelhas polinizadoras no cerrado de Botucatu, Estado de São Paulo, Brasil. Portugal Acta Biologica (A) XVII-17: 149-167. 